\title{
"Direct" Mechanical Thrombectomy in Acute Ischemic Stroke during Percutaneous Coronary Intervention
}

\author{
Vasu Saini, ${ }^{\mathrm{a}}$ Marie-Christine Brunet, ${ }^{\mathrm{b}}$ Samir Sur, ${ }^{\mathrm{b}}$ Amer M. Malik, ${ }^{\mathrm{a}}$ Priyank Khandelwal, ${ }^{\mathrm{c}}$ \\ Shashvat Desai, ${ }^{\mathrm{d}}$ Robert M. Starke, ${ }^{\mathrm{b}}$ Eric C. Peterson, ${ }^{\mathrm{b}}$ Ashutosh P. Jadhav, ${ }^{\mathrm{d}}$ Mauricio G. Cohen, ${ }^{\mathrm{e}}$ \\ Dileep R. Yavagal ${ }^{\mathrm{a}, \mathrm{b}}$ \\ aDepartment of Neurology, Jackson Memorial Hospital, University of Miami Miller School of Medicine, Miami, FL, USA \\ 'Department of Neurosurgery, Jackson Memorial Hospital, University of Miami Miller School of Medicine, Miami, FL, USA \\ 'Division of Endovascular Neurosurgery, Department of Neurosurgery, Rutgers University, Newark, NJ, USA \\ ${ }^{\mathrm{d} D i v i s i o n}$ of Interventional Neurology, Department of Neurology and Neurosurgery, University of Pittsburgh Medical Center, Pittsburgh, PA, USA \\ ${ }^{e}$ Cardiovascular Division, Department of Medicine, University of Miami Miller School of Medicine, Miami, FL, USA
}

\section{Dear Sir:}

Multiple multicenter randomized clinical trials have established mechanical thrombectomy (MT) as the standard of care (SOC) for large vessel occlusion (LVO) acute ischemic strokes (AIS). ${ }^{1}$ It is also established quite definitely that in the early thrombectomy window ( 0 to 6 hours), the neurological functional outcome is highly time-sensitive and designing workflow to minimize delays of even few minutes is critical. ${ }^{2}$ Currently, more than 1 million cardiac catheterization (CC) procedures are performed annually in the United States. The reported incidence of acute stroke during $\mathrm{CC}$ in the literature ranges from $0.1 \%$ to $0.6 \% \%^{3}$ and if a LVO stroke, can result in lifelong disability. Use of intravenous recombinant tissue plasminogen activator (IVrtPA) therapy for AIS during CC is not well established with concern for relatively higher risk of bleeding due to procedural anticoagulation. With arterial access already in place, an immediate "direct" cerebral digital subtraction angiogram (cDSA) can expedite a definitive diagnosis of LVO allowing rapid MT in such patients. Finding a LVO on immediate CDSA in a patient with acute stroke syndrome during CC makes the possibility of concurrent intracerebral hemorrhage (ICH) highly unlikely as a cause of the syndrome. A conventional head computed tomography (CT) necessitates moving the patient from the CC laboratory table to $\mathrm{CT}$ and then to neuro-angiography suite which adds significant delay to reperfusion benefit.

We hypothesized that a novel triage strategy of "direct"
cDSA, bypassing conventional head CT, followed by MT in patients who suffered a LVO AIS during CC procedures is safe and would keep the onset-to-reperfusion times short. "Direct" is defined as performing cDSA for MT in the same single-plane $\mathrm{CC}$ suite foregoing conventional $\mathrm{CT}$ to rule out $\mathrm{ICH}$. In this case series we report two large tertiary-care comprehensive stroke centers experience with this management strategy, which was agreed upon by both cardiology and neuro-endovascular teams. All direct-cDSA and MT procedures were performed by Endovascular Surgical Neuroradiology fellowship-trained neuro-interventionalists. Figure 1 highlights our institutional workflow algorithm for direct-cDSA.

Four consecutive cases were included in this case series from January 2016 to June 2017, where the acute focal neurological syndrome was identified while patients were still in the CC suite and had the arterial sheath in place. Table 1 details the baseline characteristics and covariates of patients included in this case series. All patients identified were undergoing emer-

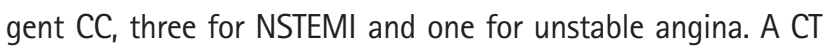
brain was obtained within 24-hour post-MT to evaluate for any hemorrhagic transformation (HT) of the infarcted cerebral tissue. LVO was confirmed in three out of four patients who subsequently underwent successful MT (Figure 2). One patient had patent vessels on direct-cDSA with rapid improvement in symptoms suggesting spontaneous recanalization or non-LVO stroke. Immediate head CT obtained in this patient did not show an ICH, and IV-rtPA was not administered because symp- 


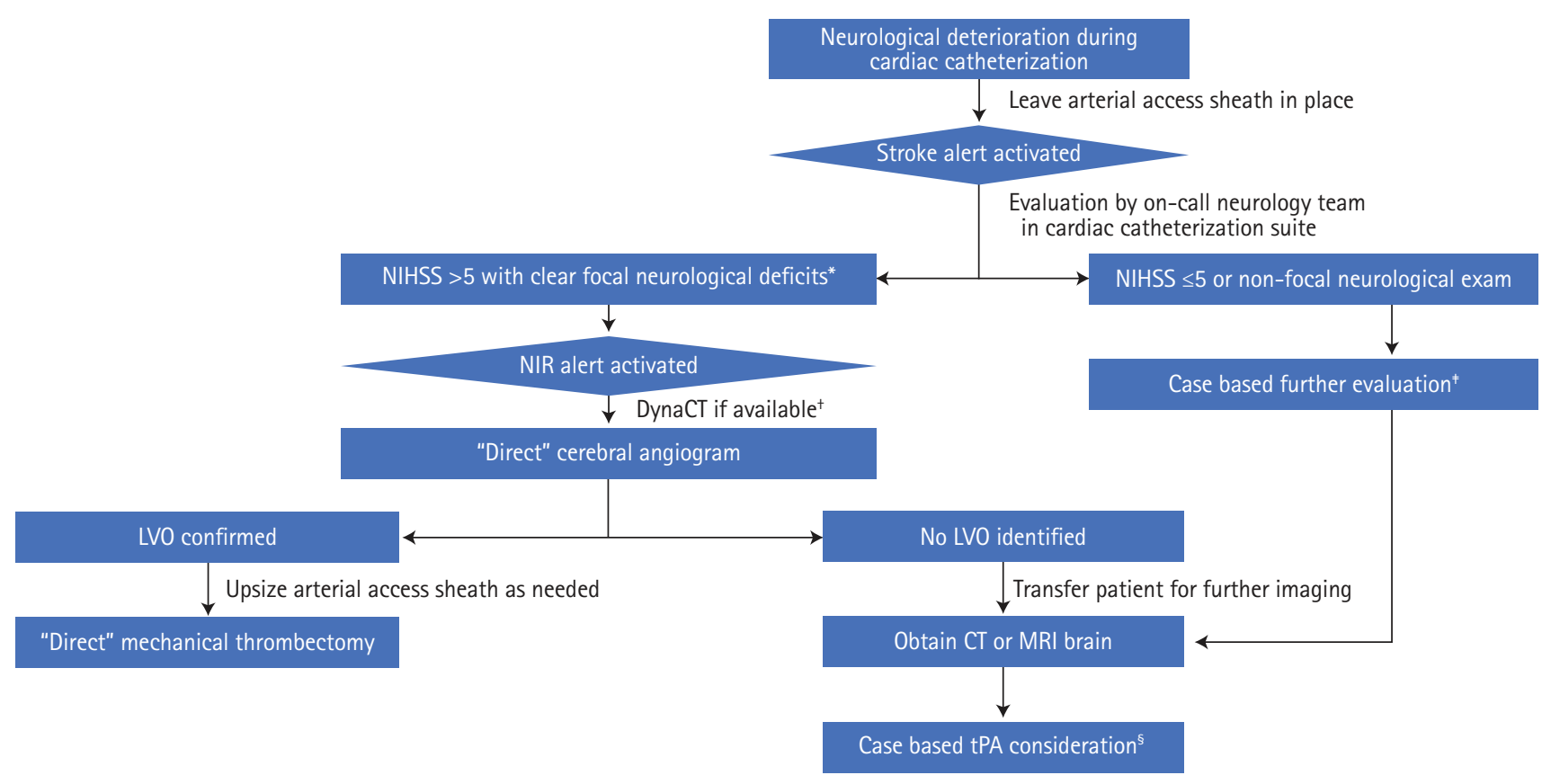

Figure 1. Algorithm to identify patients with neurological deterioration during cardiac catheterization who would benefit from direct cerebral angiogram and mechanical thrombectomy. NIHSS, National Institutes of Health Stroke Scale; NIR, neuro-interventional radiology; LVO, large vessel occlusion; CT, computed tomography; MRI, magnetic resonance imaging; tPA, tissue plasminogen activator. *Focal neurological deficits were defined as focal motor weakness or cortical deficits such as horizontal gaze deviation, hemi-visual or sensory neglect, aphasia or hemianopsia; ${ }^{+}$If cardiac catheterization suite is equipped with the newer flat-panel detector technology, a sufficient quality DynaCT (Siemens Medical Solutions) can be obtained to rule out intracranial hemorrhage, and if negative, neurointerventional team can proceed with a direct-cerebral digital subtraction angiography. This technology was not available in our cardiac catheterization suite at the time these procedures were performed; ${ }^{*}$ Cases discussed with both cardiology and neurology teams to weigh the risk and benefit of completing cardiac catheterization procedure before or after obtaining further cerebral imaging such as CT or MRI brain as needed; ${ }^{5}$ tPA can be considered after intracranial hemorrhage is ruled out and if coagulation lab tests such as prothrombin time-international normalized ratio and partial thromboplastin time are permissive for intravenous thrombolytic use per institutional guidelines.

Table 1. Baseline patient characteristics, procedural and outcome details of catheter cerebral angiography and mechanical thrombectomy

\begin{tabular}{|c|c|c|c|c|c|c|c|c|c|c|c|}
\hline & $\begin{array}{l}\text { Age (yr), } \\
\text { sex }\end{array}$ & $\begin{array}{l}\text { Clot location and } \\
\text { clinical syndrome }\end{array}$ & $\begin{array}{l}\text { Onset to } \\
\text { cDSA start } \\
(\min )^{*}\end{array}$ & $\begin{array}{l}\text { Onset to } \\
\text { first } \\
\text { pass } \\
(\mathrm{min})^{*}\end{array}$ & $\begin{array}{l}\text { Onset to } \\
\text { recanaliza- } \\
\text { tion }(\min )^{*}\end{array}$ & $\begin{array}{c}\mathrm{TICl} \\
\text { grade }^{+}\end{array}$ & $\begin{array}{l}\text { No. of } \\
\text { passes }\end{array}$ & $\begin{array}{l}\text { Initial } \\
\text { NIHSS }\end{array}$ & $\begin{array}{c}\text { 2-hr } \\
\text { post-MT } \\
\text { NIHSS }\end{array}$ & $\begin{array}{l}\mathrm{mRS} \text { at } \\
\text { discharge }\end{array}$ & $\begin{array}{l}\text { Indication for cardiac } \\
\text { catheterization }\end{array}$ \\
\hline Case 1 & $63, F$ & $\begin{array}{l}\text { L M2; near-com- } \\
\text { plete L MCA syn- } \\
\text { drome }^{\neq}\end{array}$ & 15 & 25 & 31 & 3 & 1 & 21 & 2 & 1 & $\begin{array}{l}\text { Unstable angina/NSTEMI; } \\
1 \text { coronary DES placed } \\
\text { on day } 6 \text { post-MT }\end{array}$ \\
\hline Case 2 & $69, F$ & $\begin{array}{l}\text { L M1; complete L } \\
\text { MCA syndrome }\end{array}$ & 26 & 41 & 46 & 3 & 1 & 26 & 11 & 2 & $\begin{array}{l}\text { NSTEMI; no coronary stent } \\
\text { placed }\end{array}$ \\
\hline Case 3 & $70, F$ & $\begin{array}{l}\text { R M2; complete R } \\
\text { MCA syndrome }\end{array}$ & 17 & 62 & 85 & $2 b$ & 1 & 25 & 18 & 3 & $\begin{array}{l}\text { NSTEMI; } 2 \text { coronary DES } \\
\text { placed prior to the neu- } \\
\text { rological deficit }\end{array}$ \\
\hline Case $4^{\S}$ & $50, F$ & $\begin{array}{l}\text { Suspected R M1; } \\
\text { near-complete } R \\
\text { MCA syndrome }\end{array}$ & 11 & NA & NA & NA & NA & 18 & 0 & 0 & $\begin{array}{l}\text { NSTEMI; no coronary stent } \\
\text { placed }\end{array}$ \\
\hline
\end{tabular}

cDSA, cerebral digital subtraction angiography; TICl, thrombolysis in cerebral infarction; NIHSS, National Institutes of Health Stroke Scale; MT, mechanical thrombectomy; mRS, modified Rankin Scale; L, left; M1,2, sequential divisions of MCA; MCA, middle cerebral artery; NSTEMI, non-ST elevation myocardial infarction; DES, drug-eluting stent; $R$, right; NA, not applicable.

*Onset time was defined as the time neurological deterioration was noted by cardiac catheterization team. As the arterial access sheath was already in place, time to start of CDSA procedure was defined as the first angiographic run by neurointerventional team. First pass was defined as the first attempt at clot retrieval during MT. The final angiographic run confirming the patency of previously occluded vessel after successful MT was documented as the recanalization time; 'Successful recanalization was defined as a $\mathrm{TICl}$ score of $2 b-3 ;{ }^{*}$ Complete MCA syndrome is defined as contralateral hemiplegia and hemisensory loss, ipsilateral horizontal gaze deviation, aphasia or dysarthria (in patients with involvement of dominant or non-dominant hemisphere respectively), contralateral hemianopsia and contralateral hemi-visual and/or hemi-sensory neglect (in patients with non-dominant hemisphere involvement). The severity of the syndrome is defined by NIHSS score (range 0-42); ${ }^{5}$ Vessels patent on initial cerebral angiography and therefore did not undergo MT. Symptoms improved to NIHSS 0 while the patient was being transferred for magnetic resonance imaging. 

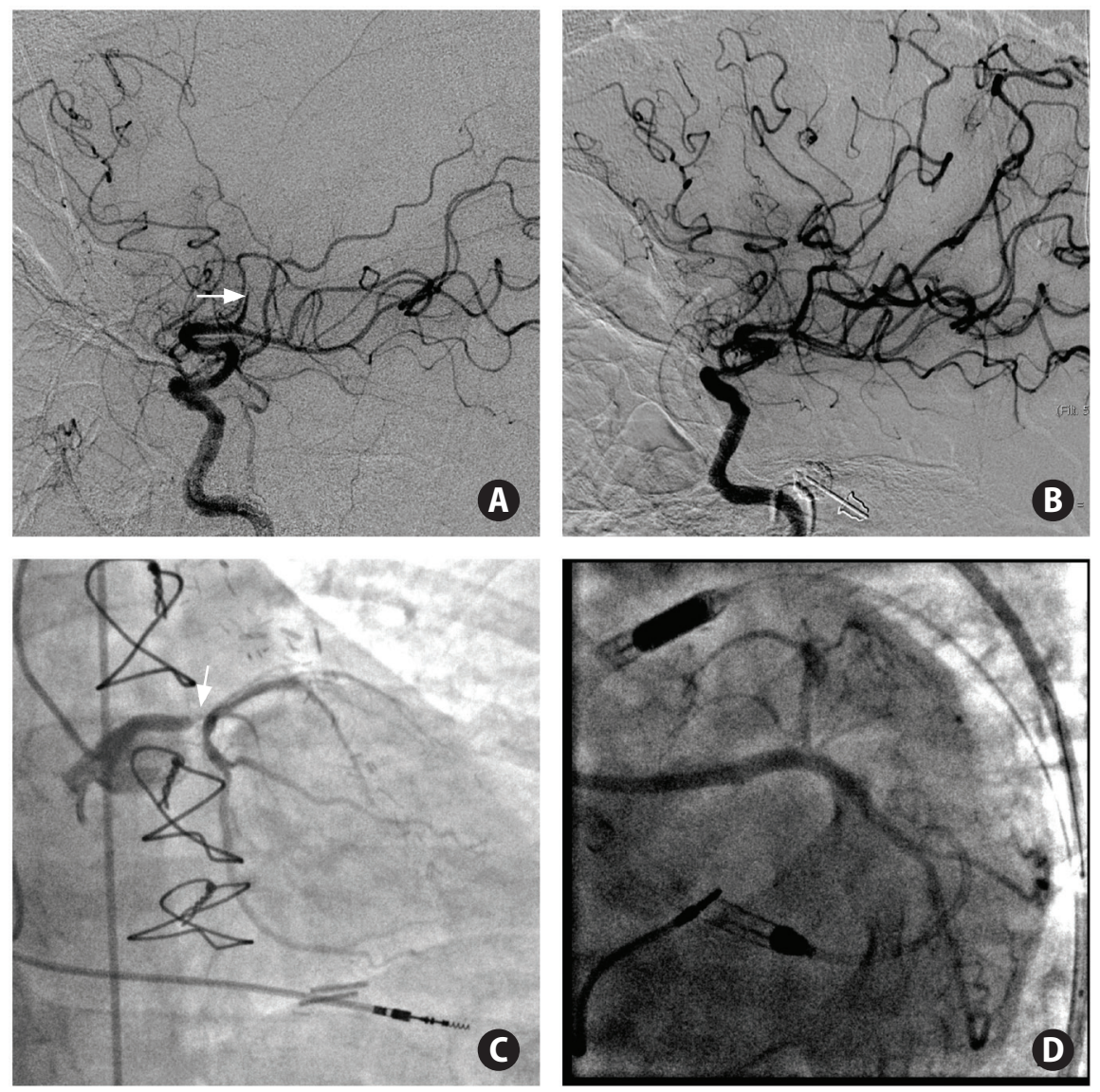

Figure 2. (A, B) Pre- and post-mechanical thrombectomy (MT) catheter cerebral angiogram images performed in the single-plane cardiac catheterization suite. (C, D). Initial diagnostic cardiac catheter angiogram on admission and percutaneous coronary intervention with a drug-eluting stent placement in left main coronary artery performed on day 6 post-MT. Case 1 (refer Table 1) underwent initial cardiac catheter angiogram showing left main coronary artery $>90 \%$ stenosis (C; white arrow), complicated by near-complete left middle cerebral artery (MCA) syndrome, and underwent immediate direct cerebral angiogram showing proximal second division of MCA respectively (M2) cut-off ( $A$; white arrow). Direct-MT performed with successful thrombolysis in cerebral infarction 3 reperfusion (B). On day 6 post-MT patient underwent successful percutaneous coronary intervention of left main coronary artery and ostial/proximal left circumflex with drug-eluting stent placement with resulting thrombolysis in myocardial infarction 3 flow.

toms spontaneously improved with return of National Institutes of Health Stroke Scale to 0. In aggregate, the mean times from the identification of neurological deficits to start of cDSA procedure, first pass and successful revascularization was 17, 43 and 54 minutes, respectively. In all cases, the same transfemoral arterial access (TFA) obtained by a cardiologist was utilized for initial cDSA with subsequent upsizing of the access sheath to $8 \mathrm{Fr}$ as needed for MT. Anticoagulation with intravenous unfractionated heparin during $C C$ procedures was used in three of four patients. IV-rtPA was not administered in any case. All CC procedures were performed under monitored anesthesia care, and none of the patients required conversion to general anesthesia for MT. Solitaire stent retriever with balloon guide catheter and standard endovascular stroke techniques were used for all MT procedures.

Since MT became SOC in 2015 and the outcomes were found to be highly time sensitive, ${ }^{1}$ a major focus is to devise time saving workflow strategies to maximize good outcomes. Our case series indicate that if a LVO is seen on CDSA in the vascular territory referable to the stroke syndrome occurring during $\mathrm{CC}$, the need for a CT to rule out hemorrhage appears to be redundant. None of the patients in our series had a HT on subsequent CT brain indicating safety of our approach. There is anecdotal evidence documenting the safety of reversal of procedural anticoagulation with IV heparin with protamine followed by successful administration of IV-rtPA for AIS during $\mathrm{CC}_{i}^{4}$ but as there is no clear consensus on this approach we opted to forego the same.

In keeping with the trend of preferred increased use of trans-radial access for cardiovascular procedures, it is important to highlight the fact that successful MT can be performed using the same radial access which can be upsized to $6 \mathrm{Fr}$ as 
needed, without the need for crossover to TFA. ${ }^{5}$ Furthermore, the angiographic suites equipped with the newer flat-panel detector technology have the capability of obtaining a sufficient quality DynaCT (Siemens Medical Solutions, Erlangen, Germany), which can be used to rule out $\mathrm{ICH}^{6}$ and if negative, would obviate the need to remove the patient from angiography table supporting our approach for direct-MT.

Our case series suggests that performing immediate direct CDSA followed by MT without conventional CT is feasible, safe and keeps the stroke onset-to-reperfusion times very short in patients with suspected LVO during CC procedures. Therefore, further and larger studies are needed to prove the use of this approach in routine clinical practice.

\section{References}

1. Goyal M, Menon BK, van Zwam WH, Dippel DW, Mitchell PJ, Demchuk AM, et al. Endovascular thrombectomy after largevessel ischaemic stroke: a meta-analysis of individual patient data from five randomised trials. Lancet2016;387:1723-1731.

2. Saver JL. Time is brain: quantified. Stroke 2006;37:263-266.

3. Werner N, Bauer T, Hochadel M, Zahn R, Weidinger F, Marco $J$, et al. Incidence and clinical impact of stroke complicating percutaneous coronary intervention: results of the Euro heart survey percutaneous coronary interventions registry. Circ Cardiovasc Interv 2013;6:362-369.
4. Warner DS, Schwartz BG, Babygirija R, Rovin RA, Kassam AB,

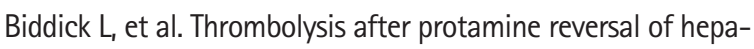
rin for acute ischemic stroke after cardiac catheterization: case report and literature review. Neurologist 2018;23:194196.

5. Chen SH, Snelling BM, Shah SS, Sur S, Brunet MC, Starke RM, et al. Transradial approach for flow diversion treatment of cerebral aneurysms: a multicenter study. J Neurointerv Surg 2019;11: 796-800.

6. Heran NS, Song JK, Namba K, Smith W, Niimi Y, Berenstein A. The utility of DynaCT in neuroendovascular procedures. AJNR Am J Neuroradiol 2006;27:330-332.

Correspondence: Dileep R. Yavagal

Department of Neurology, Jackson Memorial Hospital, University of Miami Miller School of Medicine, 1120 NW 14th St, Ste. 1383, Miami, FL 33131, USA Tel: +1-305-355-1103

Fax: +1-305-355-1102

E-mail: dyavagal@med.miami.edu

Received: February 18, 2020

Revised: May 17, 2020

Accepted: May 20, 2020

Dr. Yavagal is on the steering committee of TIGER clinical trial sponsored by Rapid Medical and steering committee of CALM-2 sponsored by Vascular Dynamics. He is a consultant to Medtronic, Cerenovus, Poseydon, Neurosave, and other Neuralanalytics. All of these relationships are outside of the current work. 